\title{
Application of Simple Harmonics Modeling a Shock
}

Kai Raymond

University of South Florida

Advisors:

Thomas McKinley, Mathematics and Statistics

Dario Arena, Physics

Problem Suggested By: Kai Raymond

Follow this and additional works at: https://digitalcommons.usf.edu/ujmm

Part of the Mathematics Commons

UJMM is an open access journal, free to authors and readers, and relies on your support:

Donate Now

\section{Recommended Citation}

Raymond, Kai (2017) "Application of Simple Harmonics Modeling a Shock," Undergraduate Journal of Mathematical Modeling: One + Two: Vol. 8: Iss. 1, Article 1.

DOI: http://doi.org/10.5038/2326-3652.8.1.4882

Available at: https://digitalcommons.usf.edu/ujmm/vol8/iss1/1 


\title{
Application of Simple Harmonics Modeling a Shock
}

\begin{abstract}
This project entails modeling a spring-shock absorber system in order to evaluate the position of a mass when this system is acted upon by outside forces. We will restrict our attention to the linear case where it is assumed that Hooke's law gives the force exerted by the spring, and the resistance of the system will be proportional to the velocity of the mass. We analyze the time and frequency domain of the absorber system and compute its impulse response.
\end{abstract}

\section{Keywords}

spring systems, Hooke's law, overdamped systems, underdamped systems, impulse response

\section{Creative Commons License}

(c) (i) $9 \Theta$

This work is licensed under a Creative Commons Attribution-Noncommercial-Share Alike 4.0 License. 


\section{PROBLEM STATEMENT}

A spring whose restoring force is proportional to its position with respect to the equilibrium position is acted upon by an external force. While being in motion on a surface, the surface creates a friction whose magnitude is proportional to the speed of the spring at any time. The free body diagram of the spring is considered and Newton's second law gives a second order linear differential equation with constant coefficients in the position as a function of time together with its first and second derivatives. Depending on the system parameters, three different solutions are considered with the impulse response in each single case.

\section{MOTIVATION}

Wanting to know the behavior of a spring shock assembly of a dirt bike had motivated this project. There are several adjustments that can be made on such an assembly and I wanted to be able to predict its behavior as a result of such adjustments.

\section{MATHEMATICAL DESCRIPTION AND SOLUTION APPROACH}

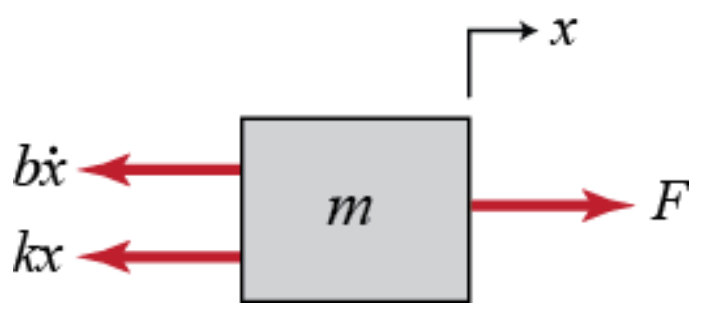

Figure 1: Schematic of the forces acting on the dirt bike spring shock assembly where $k x$ is the force exerted by the spring, $b \dot{x}$ is the friction for and $F$ is an external force.

Figure 1 indicates the basic forces acting on the mass with respect to time and displacements. Using Newton's second law, we have 


$$
F_{n e t}=F-\left(b \frac{d x}{d t}+k x\right)=\left(m \frac{d^{2} x}{d t^{2}}\right)-\left(b \frac{d x}{d t}+k x\right)
$$

Re-writing (1) we have the following second order linear differential equation;

$$
\frac{d^{2} x}{d t^{2}}+\frac{b}{m} \frac{d x}{d t}+\frac{k}{m}=\frac{F_{n e t}}{m}
$$

where $x$ is displacement, $b$ is resistance and $k$ is the spring constant in eqs. (1) and (2) here, [3]. The homogeneous form of (2) is

$$
\frac{d^{2} x}{d t^{2}}+\frac{b}{m} \frac{d x}{d t}+\frac{k}{m}=0
$$

and the general solution to (2) is given by

$$
x(t)=x_{h}(t)+x_{p}(t)
$$

where $x_{h}(t)$ is the general solution of (3), i.e., the homogeneous solution, and $x_{p}(t)$ is the particular solution which depends on $F_{\text {net }}$. The homogeneous solution has the following forms,[1],[3]:

i. $\quad x_{h}(t)=C_{1} e^{a t}+C_{2} e^{b t}$ where $a \neq b$

ii. $\quad x_{h}(t)=C_{1} e^{a t}+C_{2} t e^{a t}$

iii. $\quad x_{h}(t)=C_{1} e^{(\alpha+i \beta) t}+C_{2} e^{(\alpha-i \beta) t}$

Here for the homogenous solution of the form, $C_{1} e^{(\alpha+i \beta) t}+C_{2} e^{(\alpha-i \beta) t}$, the constants are complex conjugates for the case involving real solutions. Of course this is all we are interested in having.

If $F(t)$, the forcing function, has the form $A \cos (\omega t)$ with constants $A$ and $\omega$, then

$$
F_{p}(t)=B_{1} \cos (\omega t)+B_{2} \sin (\omega t)=C \cos (\omega t+\varphi)
$$

for some contants $B_{1}, B_{2}, C$, and $\varphi$. As $t$ tends to infinity, the general solution above tends to the particular solution, $F_{p}(t)$. This is what is often called the steady state solution. $C(\omega)$ and $C(\varphi)$ 
refer to the magnitude and phase response with respect to frequency. This will be one of the evaluations that are made in what's to come.

For the homogeneous solution in (3), there are 3 categories of response as already indicated by (i), (ii), and (iii). These cases depend on the roots of the characteristic equation given by,

$$
r^{2}+\frac{b}{m} r+\frac{k}{m}=0
$$

If the roots are real and distinct we have (i), and these roots are $a$ and $b$ respectively. When this occurs we say the system is overdamped. If the root is a repeated root, then we have case (ii), and $a$ is this root.

Finally, if the roots of (6) are complex conjugates, $(\alpha \pm i \beta)$, we have case (iii). For this case, we say the system is underdamped. See the following Figures 2,3 and 4.
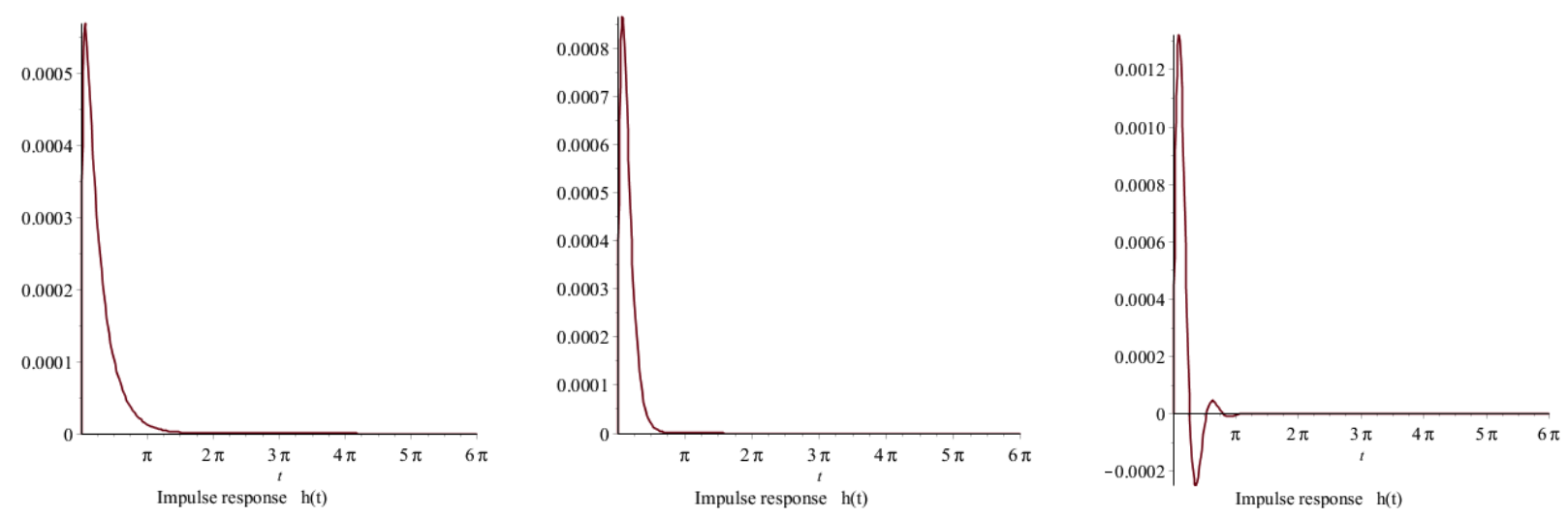

\section{DISCUSSION}

As part of the analysis to follow we will evaluate the Dirac delta impulse response. This is the response of the system with $\delta(t)$ as input. The impulse response will be denoted, $h(t)$. 
This response indicates whether the system is underdamped, critically damped, or underdamped. The system behavior to any input can be found by convoluting $h(t)$ with that input.

In order to solve the system we will take the Laplace transform of the second order linear differential equation from above which Pierre Laplace stated that solutions of this equation are called Harmonic functions [4]. The Laplace transform of eq. (2) was solved for and obtained

$$
X(s)=\frac{F_{n e t}(s)}{s^{2}+\frac{b}{m} s+\frac{k}{m}}
$$

$X(s)$ is the Laplace transform of the system output response and $F(s)$ is the input [1]. The impulse response $h(t)$ can be obtained from

$$
h(t)=\mathcal{L}^{-1}\left(\frac{1}{s^{2}+\frac{b}{m} s+\frac{k}{m}}\right)
$$

[2],[3]. The system transfer function is defined by

$$
\frac{X(s)}{F_{n e t}(s)}=\frac{1}{s^{2}+\frac{b}{m} s+\frac{k}{m}}
$$

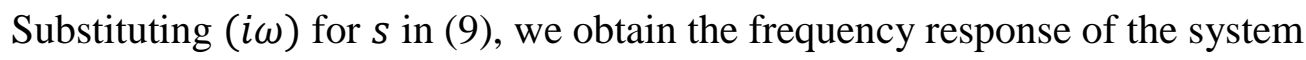

$$
F(\omega)=\frac{1}{\left(\frac{k}{m}-\omega^{2}\right)+i \frac{b}{m} \omega}
$$

The frequency response was discussed earlier before equation (5). Eq (10) gives the response of the system when $F_{n e t}=\cos (\omega t)$. The steady state output, $x(t)=C \cos (\omega t+\varphi)$, where

$$
C=|F(\omega)| \quad \text { and } \quad \varphi=\operatorname{Arg}(F(\omega)) .
$$


Maple 18 was used to evaluate $h(t)$, the output due to a single pulse input, the output due to a pulse train input, and the frequency response[1],[3]. These evaluations span over several cases as indicated in the experiment / conclusion section.

\section{CONCLUSION AND RECOMMENDATIONS}

The pulse input and pulse train inputs that will be used in the following four cases are shown in the following figures:

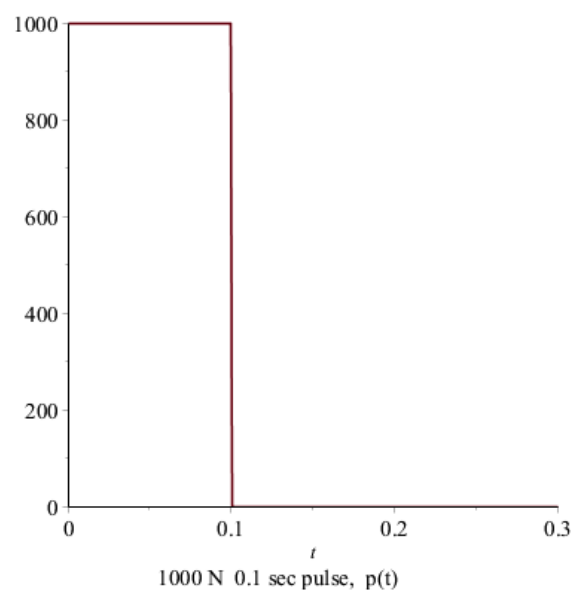

Figure 5: Single pulse force of $1000 \mathrm{~N}$ having a duration of $0.1 \mathrm{sec}$.

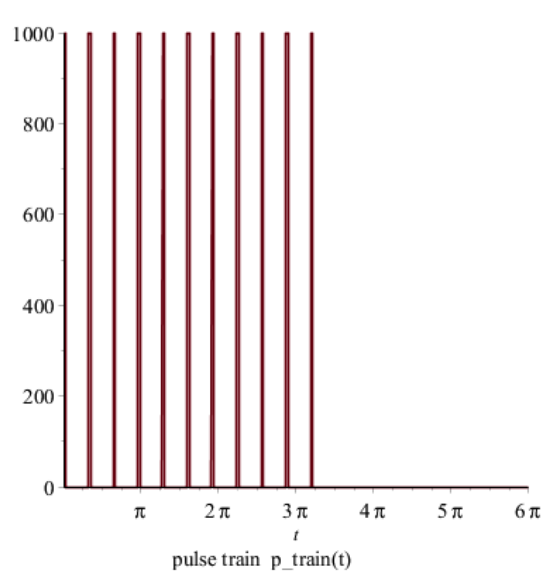

Figure 6: A train of 10 pulses. Each pulse is a replica of the pulse shown in Figure 5. The pulses are spaced one second apart.

In the cases to follow, $b$ indicates the resistance in $N \frac{s}{m} . k$ is the spring constant in $\frac{N}{m}$. The mass $m$ is in kilograms $(\mathrm{kg})$.

Case 1: Heavily Underdamped

$m:=100: \quad$ \#mass of bike and rider $\mathrm{kg}$.

$b:=100:$ \#resistance of shock $N \cdot \frac{s}{m}$.

$k:=1800: \quad$ \# spring constant $\frac{N}{m}$.

The impulse of this system is shown below,[2] 


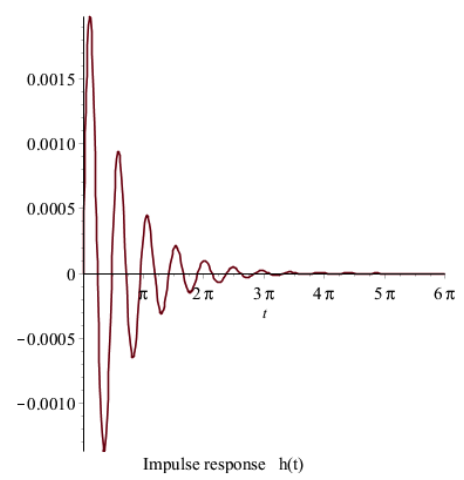

Note the ringing in this case.

The systems response to the single pulse is shown next

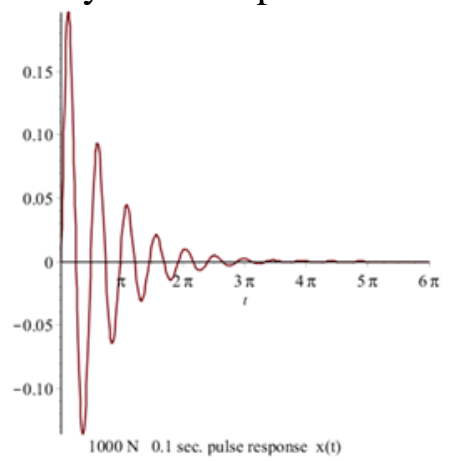

The response of the system due to the pulse train is illustrated below

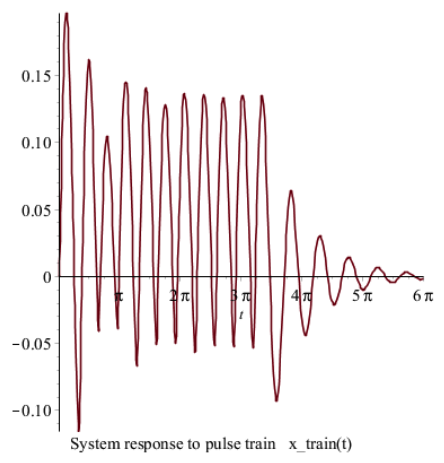

Note the response of the heavily underdamped system due to the pulse train oscillates greatly about the equilibrium position.

Finally, the frequency response (magnitude and phase) are shown next. 

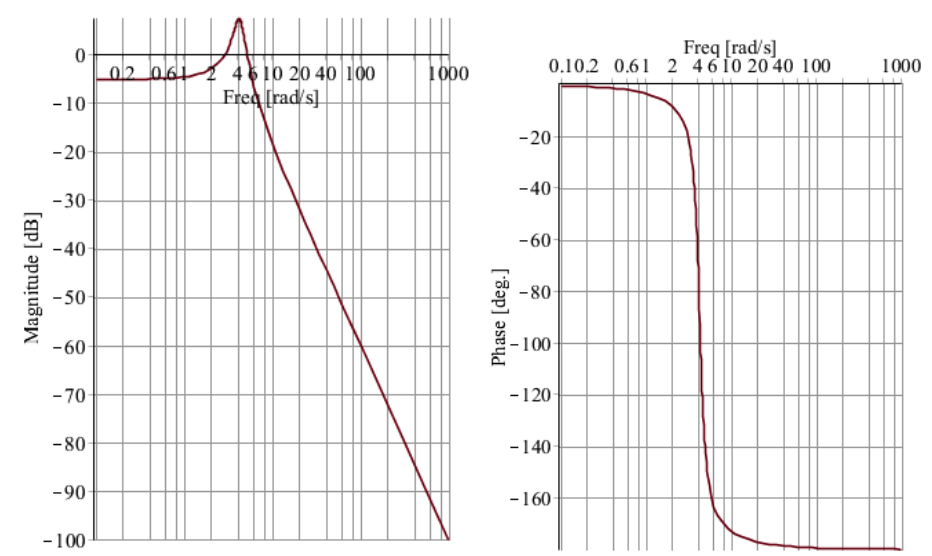

Note the magnitude (the left figure) has a peak at about the resonance frequency for the system. This is to be expected for the heavily underdamped case.

Case 2: Underdamped

$$
\begin{aligned}
& m:=100: \quad \text { \#mass of bike and rider } \mathrm{kg} . \\
& b:=400: \text { \#resistance of shock } N \cdot \frac{\mathrm{s}}{m} . \\
& k:=1800: \quad \text { \# spring constant } \frac{N}{m} .
\end{aligned}
$$

The impulse response is shown below.

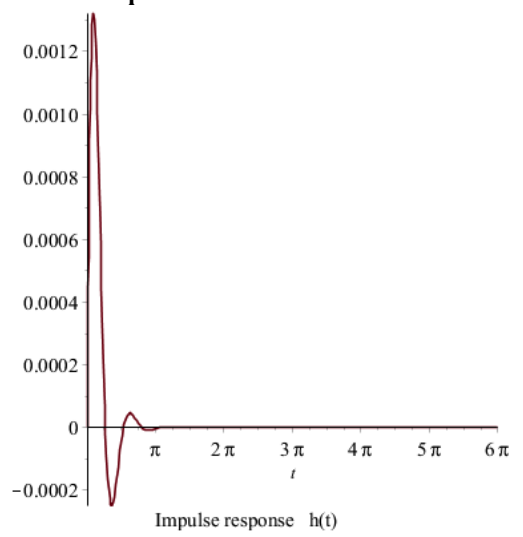

The ringing is far less than in the first case where the system is heavily underdamped,[2].

The figure shown on the next page is the systems response to a pulse. 


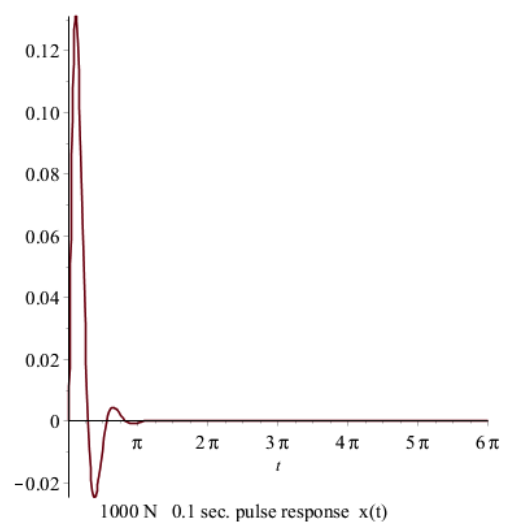

The next figure shows the response of this system to the pulse train.

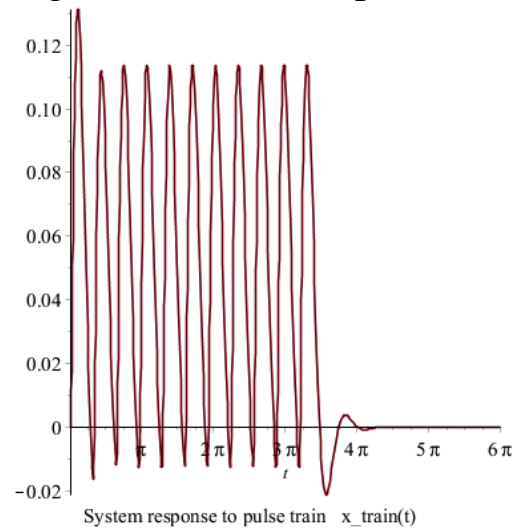

Note the amplitude of the oscillations here is far less than in the first case. Also, since the force of the pulse train is positive, the equilibrium about these oscillations is positive in position.

Next the frequency response of the system is given;
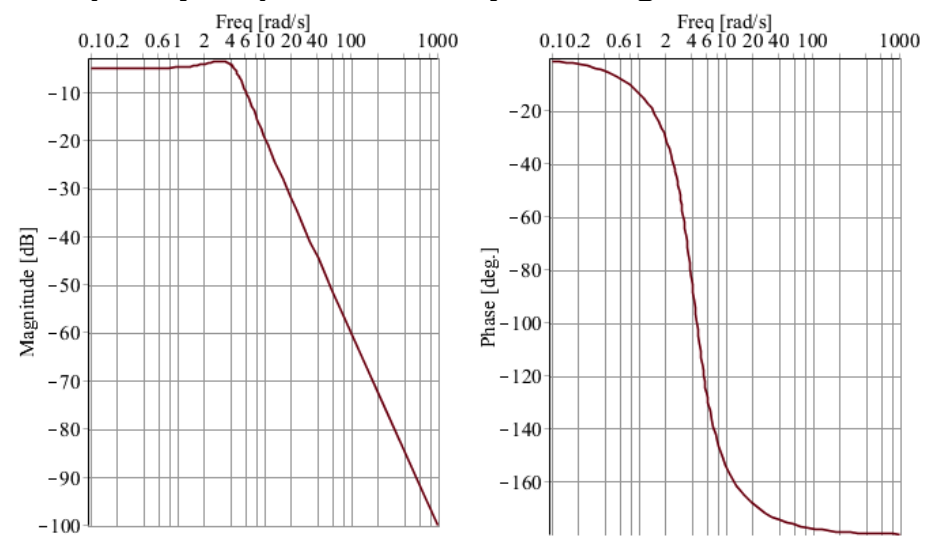

Note that the magnitude response (left) still has a small peak near the resonance frequency.

Case 3: Critically Damped 


$$
\begin{aligned}
& m:=100: \quad \text { \#mass of bike and rider } \mathrm{kg} . \\
& b:=848.6: \text { \#resistance of shock } N \cdot \frac{\mathrm{s}}{m} . \\
& k:=1800: \quad \text { \# spring constant } \frac{N}{m} .
\end{aligned}
$$

The impulse response is shown below.

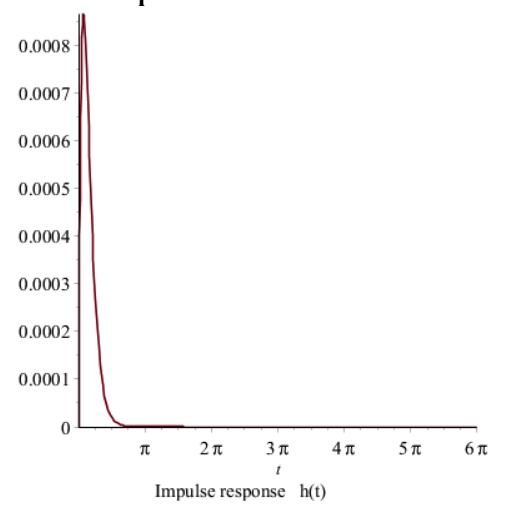

Note that the oscillations have disappeared here,[2].

Next the pulse response is given.

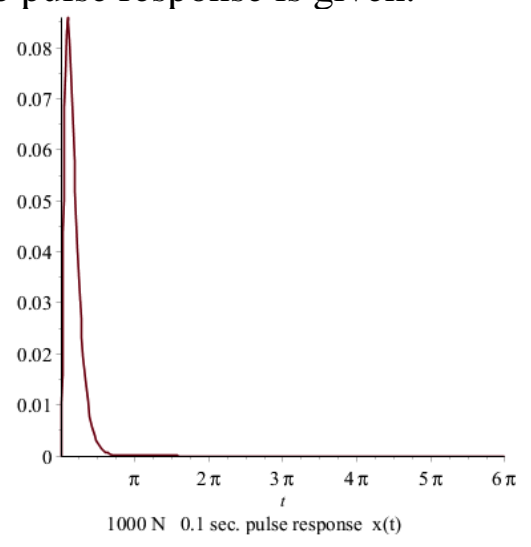

Next the response to the pulse train is given. Here the oscillations due to the input are about a positive displacement of the spring. 


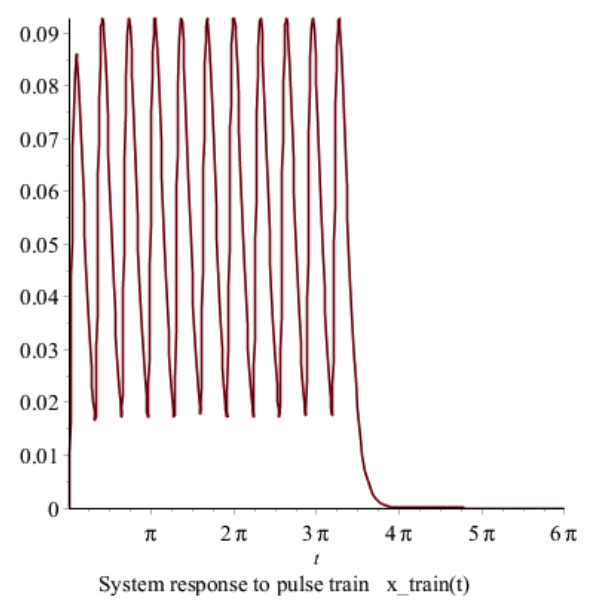

Finally, we show the frequency response of this system.
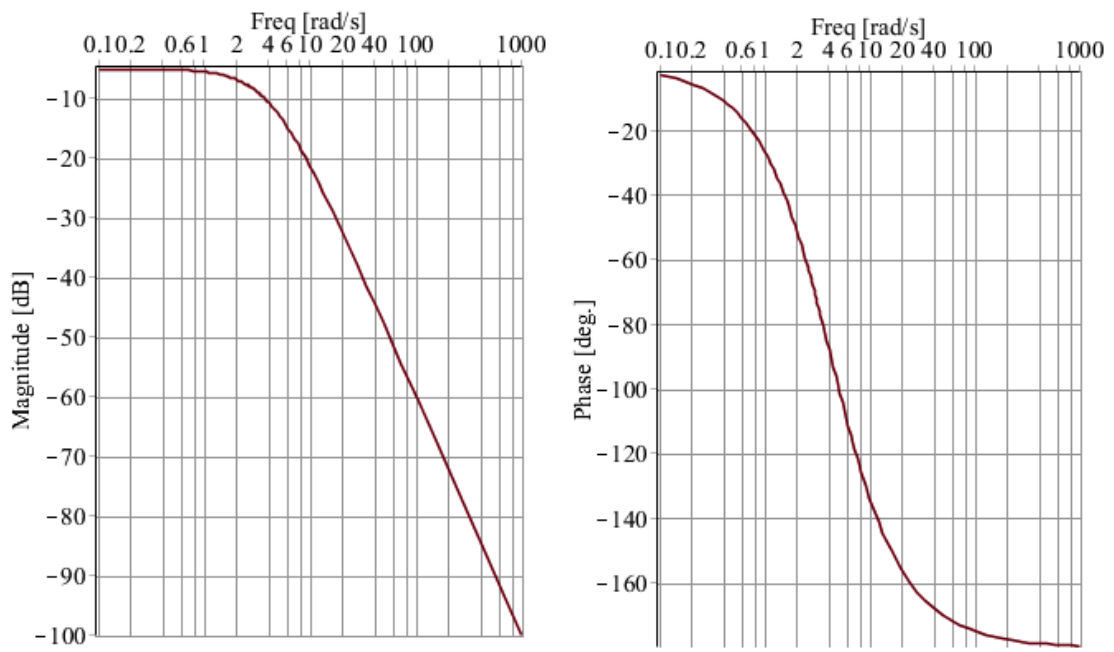

Note there is no peak in the magnitude response (left). This has the appearance of a "second order Butterworth filter". [3]

Case 4: Overdamped

$$
\begin{aligned}
& m:=100: \quad \text { \#mass of bike and rider } \mathrm{kg} . \\
& b:=1500: \text { \#resistance of shock } N \cdot \frac{\mathrm{s}}{m} . \\
& k:=1800: \quad \text { \# spring constant } \frac{N}{m} .
\end{aligned}
$$

The impulse response for this case is shown next. There are no oscillations. The decay is purely exponential,[2]. 


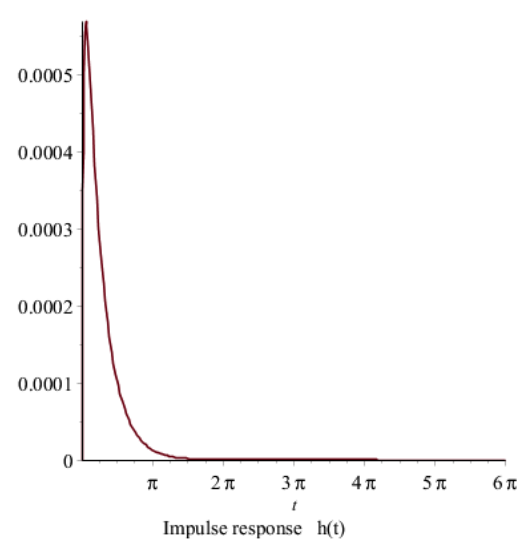

The next figure shows the response of this system to the pulse input.

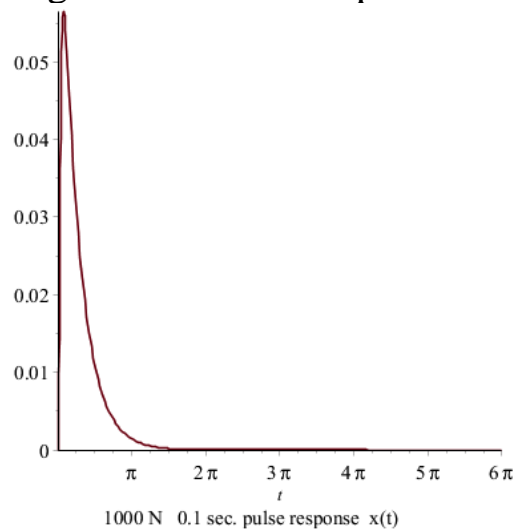

Next the systems response to the pulse train is given.

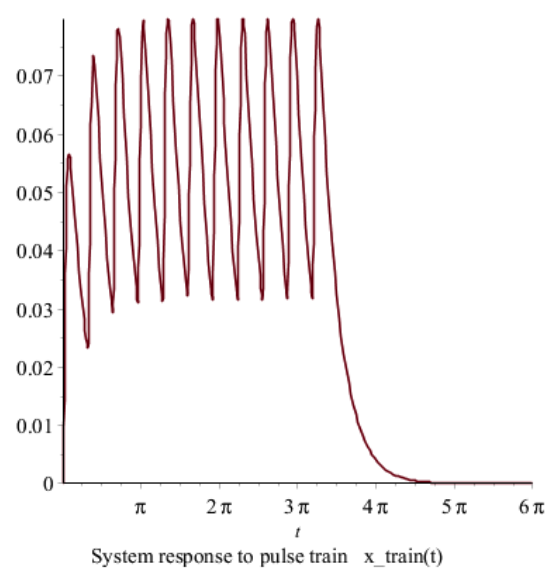

The remaining figures indicate the magnitude and phase response of the system. 

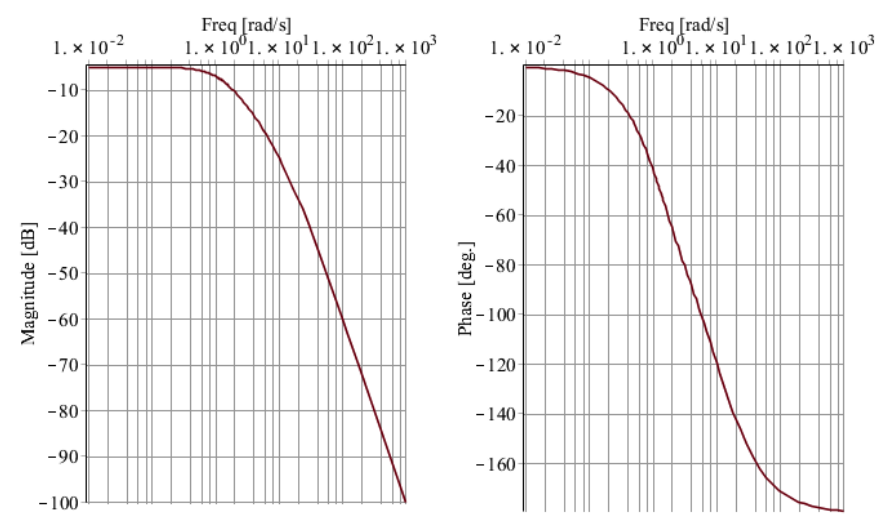

The results given in the cases were expected. The two underdamped cases showed oscillations in the impulse response and more so in the heavily underdamped case. Their frequency responses showed a peak at or near the resonance frequency as expected.

For the critically damped and overdamped cases, the impulse responses showed no oscillation. The magnitude of their frequency responses gave no peak at the resonance as expected.

\section{NOMENCLATURE}

\begin{tabular}{clc}
\hline Symbol & \multicolumn{1}{c}{ Description } & Value \\
\hline \hline$\pi$ & Apple Pie & 8 slices \\
$\vartheta$ & What's a henweigh? & $0.9 \mathrm{~kg}$ \\
$G$ & Gravitational constant & $6.67408 \times 10^{-11} \frac{\mathrm{m}^{3}}{\mathrm{~kg} \mathrm{~s}^{2}}$ \\
\hline
\end{tabular}

\section{REFERENCES}

[1] Haynes Miller, and Arthur Mattuck. 18.03 Differential Equations. Spring 2010.

Massachusetts Institute of Technology: MIT OpenCourseWare, https://ocw.mit.edu.

License: $\underline{\text { Creative Commons BY-NC-SA. }}$

[2] Kahol, P., \& Foster, D. (2004). Physics for scientists and engineers, a strategic approach:

Randall D. Knight. San Francisco, Ca.: Pearson/Addison Wesley. 
[3] Proakis, J. G., \& Salehi, M. (2005). Fundamentals of communication systems. Upper Saddle River, NJ: Pearson Prentice Hall.

[4] Stewart, J. (2013). Essential calculus: early transcendentals. Belmont, CA: Brooks / Cole, Cengage Learning. 